Argonne

\title{
Performance of Compact Pulsed Thermal Imaging System for In-Service Applications
}

Pulsed Thermal Tomography Nondestructive Examination of Additively Manufactured Reactor Materials and Components

Nuclear Science and Engineering Division 


\title{
About Argonne National Laboratory
}

Argonne is a U.S. Department of Energy laboratory managed by UChicago Argonne, LLC under contract DE-AC02-06CH11357. The Laboratory's main facility is outside Chicago, at 9700 South Cass Avenue, Argonne, Illinois 60439. For information about Argonne and its pioneering science and technology programs, see www.anl.gov.

\section{Document availability}

Online Access: U.S. Department of Energy (DOE) reports produced after 1991 and a growing number of pre-1991 documents are available free at OSTI.GOV

(http://www.osti.gov/), a service of the U.S. Dept. of Energy's Office of Scientific and

Technical Information

\author{
Reports not in digital format may be purchased by the public from the \\ National Technical Information Service (NTIS): \\ U.S. Department of Commerce \\ National Technical Information Service \\ 5301 Shawnee Rd \\ Alexandria, VA 22312 \\ www.ntis.gov \\ Phone: (800) 553-NTIS (6847) or (703) 605-6000 \\ Fax: (703) 605-6900 \\ Email: orders@ntis.gov
}

\author{
Reports not in digital format are available to DOE and DOE contractors from the \\ Office of Scientific and Technical Information (OSTI): \\ U.S. Department of Energy \\ Office of Scientific and Technical Information \\ P.O. Box 62 \\ Oak Ridge, TN 37831-0062 \\ www.osti.gov \\ Phone: (865) 576-8401 \\ Fax: (865) 576-5728 \\ Email: reports@osti.gov
}

Disclaimer

This report was prepared as an account of work sponsored by an agency of the United States Government. Neither the United States Government nor any agency thereof, nor UChicago Argonne, LLC, nor any of their employees or officers, makes any warranty, express or implied, or assumes any legal liability or responsibility for the accuracy, completeness, or usefulness of any information, apparatus, product, or process disclosed, or represents that its use would not infringe privately owned rights. Reference herein to any specific commercial product, process, or service by trade name, trademark, manufacturer, or otherwise, does not necessarily constitute or imply its endorsement, recommendation, or favoring by the United States Government or any agency thereof. The views and opinions of document authors expressed herein do not necessarily state or reflect those of the United States Government or any agency thereof, Argonne National Laboratory, or UChicago Argonne, LLC. 


\section{Performance of Compact Pulsed Thermal Imaging System for In- Service Applications}

Pulsed Thermal Tomography Nondestructive Examination of Additively Manufactured

Reactor Materials and Components

prepared by

Alexander Heifetz ${ }^{1}$, Xin Zhang $^{1,2}$, Jafar Saniie ${ }^{2}$, Sasan Bakhtiari ${ }^{1}$

${ }^{1}$ Nuclear Science Engineering Division, Argonne National Laboratory

${ }^{2}$ Department of Electrical and Computer Engineering, Illinois Institute of Technology, Chicago, IL

March 31, 2021 


\section{Table of Contents}

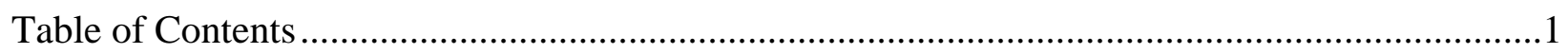

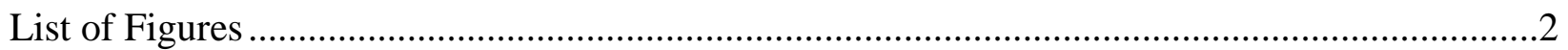

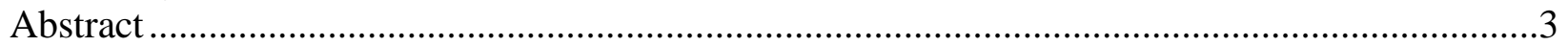

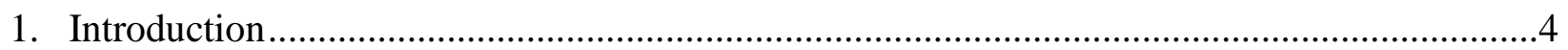

2. Pulsed Thermography Imaging System ...................................................................6

3. Sparse Coding with Discrete Cosine Transform (SC/DCT) Algorithm for Removal of Additive

White Gaussian Noise from Thermal Images ...............................................................8

4. Spatial Temporal Denoised Thermal Source Separation (STDTSS) Machine Learning

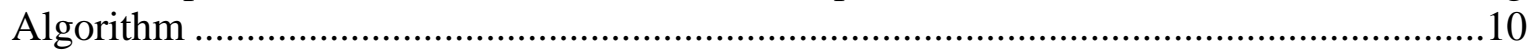

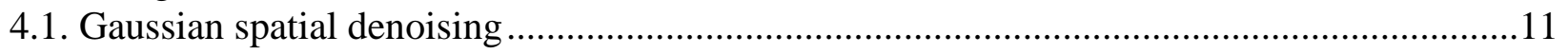

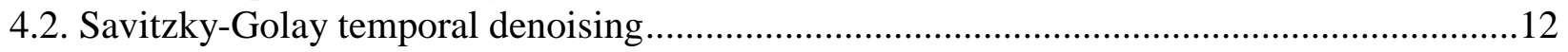

4.3. Neural learning-based matrix decomposition ...............................................................12

5. Detection of Imprinted Calibrated Defects in SS316L AM Plate with STDTSS Algorithm ...14

5.1. Development of SS316L additively manufactured specimens ..........................................14

5.2. Detection of larger defects with STDTSS algorithm .................................................15

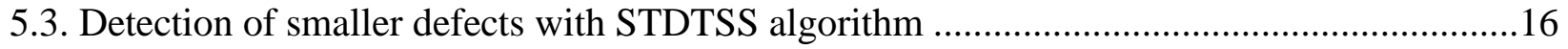

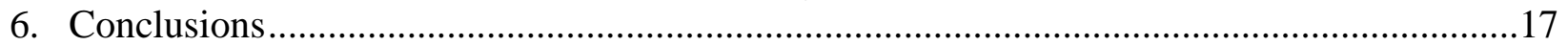

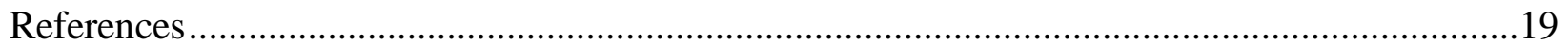




\section{List of Figures}

Figure 1 - Flash thermography experimental setup: (a) Schematic drawing (b) Photograph of actual laboratory system using compact A65 FLIR camera.

Figure 2 - (Left) Laboratory PTT system using high-end IR camera. (Right) Reconstructed effusivity e(x,y) at $\mathrm{z}=1 \mathrm{~mm}$ depth

Figure 3 - (Left) Laboratory PTT system using compact lower-cost IR camera. (Right) Reconstructed effusivity e(x,y) at $\mathrm{z}=1 \mathrm{~mm}$ depth processed with SC/DCS algorithm................... 9

Figure 4 - Flowchart of STDTSS algorithm................................................................... 11

Figure 5 - (Top) 3D rendering of hemispherical porosity defects imprinted in AM specimens. (Bottom) Design pattern of hemispherical defects of different diameters and depths relative to plate

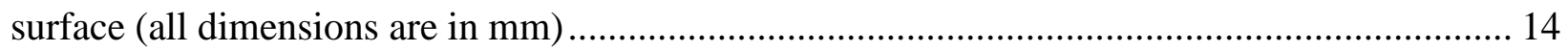

Figure 6 - Results of detection of larger defects in SS316L specimen. (a) Area of the plate (b) Raw thermogram (c) Estimated TSI with STDTSS................................................................ 15

Figure 7 - Results of detection of smaller defects in specimen SS316L specimen. (a) Area of the plate (b) Raw thermogram (c) Estimated TSI with STDTSS. 16 


\section{Abstract}

Additive manufacturing (AM) is an emerging method for cost-efficient fabrication of complex topology nuclear reactor parts from high-strength corrosion resistance alloys, such as stainless steel and Inconel. AM of metallic structures for nuclear energy applications is currently based on laser powder bed fusion (LPBF) process, which has the capability of melting metallic powder and net shaping the structures with relatively high precision. Some of the challenges with using LPBF method for nuclear manufacturing include the possibility of introducing pores into metallic structures. Integrity of AM structures needs to be evaluated nondestructively because material flaws could lead to premature failures due to creep in high temperature nuclear reactor environment. Currently, there exist limited capabilities to evaluate actual AM structures nondestructively. Pulsed Thermography (PT) imaging provides a capability for non-destructive evaluation (NDE) of sub-surface defects in arbitrary size structures. The PT method is based on recording material surface temperature transients with infrared (IR) camera following thermal pulse delivered on material surface with flash light. The PT method has advantages for NDE of actual AM structures because the method involves one-sided non-contact measurements and fast processing of large sample areas captured in one image. The data cube of PT measurements consists of surface temperature taken at sequential time intervals $\mathrm{T}(\mathrm{x}, \mathrm{y}, \mathrm{t})$. Material defects can be detected either by analyzing the thermograms $\mathrm{T}(\mathrm{x}, \mathrm{y}, \mathrm{t})$ data cube, or by using thermal tomography (TT) algorithm to obtain 3D spatial reconstruction of thermal effusivity e $(x, y, z)$.

To reduce the cost and enable in-service NDE in spatially constrained environment, it is highly desirable to develop PT with compact and inexpensive IR camera. Following initial qualification of an AM component for deployment in a nuclear reactor, a compact PT system can also be used for in-service nondestructive evaluation (NDE) applications. However, data cube obtained with PT based on compact IR camera suffers from strong thermal noises and loss of features due to relatively low sampling rate. In this report we describe two unsupervised machine learning (ML) algorithms for enhancement of PT images obtained with compact IR camera. In one approach, we introduce Sparse Coding Discrete Cosine Transform (SC/DCT) algorithm to remove additive white Gaussian noise (AWGN) from spatial thermal effusivity reconstructions. In another approach we introduce a Spatial Temporal Denoised Thermal Source Separation (STDTSS) ML algorithm to process thermograms. The STDTSS algorithm consists of spatial and temporal denoising using Gaussian and Savitzky-Golay filtering, followed by the matrix decomposition using Principal Component Analysis (PCA), and Independent Component Analysis (ICA) to automatically detect flaws.

In the work described in this report, we constructed a compact PT system using a relatively small and low-cost FLIR A65 camera, consisting on uncooled microbolometer detector. Performance of SC/DCT algorithm was demonstrated on enhancing TT images of Inconel 718 AM plate. Performance of the STDTSS methods was investigated using thermography data obtained from imaging stainless steel $316 \mathrm{~L}$ specimens produced with LPBF method with imprinted calibrated porosity defects. 


\section{Introduction}

Additive manufacturing (AM) of metals is an emerging method for cost-efficient production of low volume custom structures for industries, such as nuclear energy [1]. Metals of interest for nuclear applications typically include high-strength corrosion-resistant alloys, such as stainless steel 316L (SS316L) and Inconel 718 (IN718). Because of high melting temperature, AM of SS316L and IN718 is currently based on laser powder-bed fusion (LPBF) process [2]. Due to the intrinsic features of LPBF process, pores can appear in 3D printed metallic structures [2]. With the exposure to high temperature and creep damage in high-temperature nuclear reactors, a pore can potentially become a seed for cracking [3]. Because of stringent safety requirements, each AM metallic structure needs to be qualified through nondestructive examination before deployment in a nuclear reactor [4]. If a defect is discovered, either a flaw mitigation with heat treatment can be performed, or the part could be disqualified from service.

Typical porosity defects observed in LPBF manufacturing consist of spheroidal-shape keyhole pores caused by excessive laser power, irregular-shape lack of fusion (LOF) pores caused by insufficient laser power, and spherical gas pores caused by trapped of gas in solidifying melt pool. Sizes of pores depend on the quality of the LPBF process. Typical sizes of keyhole and LOF pores in tens to hundreds of microns, while gas pores sizes are on the order of a few microns [5]. Prior studies indicate that larger size pores located closer to surface are more likely to cause fatigue crack initiation. High-resolution imaging with X-ray or neutron computed tomography (CT) can be used for imaging pores in small coupons to evaluate quality of LPBF process. However, applications of X-ray or neutron CT to NDE of actual AM structures are limited because of large size, lack of symmetry and complex shapes of AM structures. Ultrasonic testing is scalable with structure size, but face challenges because the rough surfaces, characteristic of AM structures, affect the probe coupling. For high-resolution ultrasonic tomography, imaging of large structures is time-consuming because of point-by-point raster scanning of specimens [6].

We investigate Pulsed Thermography (PT) imaging for NDE of AM structures. This method offers several potential advantages because PT measurements are one-sided, non-contact and scalable to arbitrary size structures [7]. The PT method consists of recording material surface temperature transients with an infrared (IR) camera, following deposition of thermal impulse on material surface with a flash lamp. Material defects can be detected by analyzing thermograms (surface temperatures $\mathrm{T}(\mathrm{x}, \mathrm{y})$ ) in the data cube of sequentially recorded $\mathrm{T}(\mathrm{x}, \mathrm{y}, \mathrm{t})[4,8]$. Alternatively, 3D spatial effusivity e $(\mathrm{x}, \mathrm{y}, \mathrm{z})$ can be reconstructed from $\mathrm{T}(\mathrm{x}, \mathrm{y}, \mathrm{t})$ using thermal tomography (TT) algorithm $[5,9,10,11]$.

In prior studies, we have demonstrated PT performance in detection of calibrated imprinted defects in SS316L specimens produced with LPBF method. In general, high resolution imaging in PT requires expensive IR cameras with low-noise cooled semiconductor detectors [4]. The cost of NDE system adds the overall cost of AM process. In addition to cost considerations, the size of the high-end camera places constrains on the application of PT in in-service NDE in spatially constrained environment [5]. Therefore, it is highly desirable to develop a PT system based on compact and inexpensive IR camera. However, a compact lower-cost IR camera, typically 
consisting of uncooled microbolometer detector array, suffers from stronger thermal noise, and offers lower sensitivity, and lower frame rate. These performance characteristics limit compact PT system capability in detection of defects in AM structures.

Advances in machine learning (ML) and image processing can potentially compensate for reduced performance characteristics in IR imaging sensor hardware [5]. In this report, we investigate two algorithmic strategies for processing PT images obtained with a compact IR camera. In one approach, we investigate ML algorithms that perform de-noising function without introducing blur into images. In a recent work, we have investigated de-noising thermal images using additive white Gaussian noise (AWGN) model of IR camera noise. We developed Sparse Coding-related K-means Singular Value Decomposition (SC/K-SVD) ML algorithm for removing AWGN from PT images obtained with compact IR camera without blurring the images [5]. In this report, we describe an alternative algorithm based on Sparse Coding and Discrete Cosine Transform (SC/DCT) for removing AWGN. We demonstrate performance of SC/DCT in efficient removing AWGN from TT images of an AM IN718 plate.

As a more general strategy, we investigate ML algorithms capable of model-agnostic denoising and feature detection in thermal images. Neural Learning Blind Source Separation (NLBSS), spatial-temporal blind source separation (STBSS) and spatial-temporal sparse dictionary learning (STSDL) methods which we developed recently, enhance detection of features a data-efficient manner, but performs best with lower-noise high-end IR camera [4,8]. Pulsed Phase Thermography (PPT) [12], can improve feature detection in frequency spectra using Discrete Fourier Transform but is vulnerable to high-frequency noise [13]. In this report, we describe development of Spatial Temporal Denoised Thermal Source Separation (STDTSS) ML algorithm for processing PT images obtained with a compact IR camera, which includes de-noising and feature detection [14]. The STDTSS involves spatial and temporal denoising using Gaussian filtering [15] and Savitzky-Golay filtering [16], followed by the matrix decomposition using Principal Component Analysis (PCA) [15], and Independent Component Analysis (ICA) [17], to automatically detect flaws in images. In STDTSS, a 2D Gaussian filter is designed to spatially denoise high frequency components in thermography images, while preserving signatures of material defects. In addition, a 7-point Savitzky-Golay filter is utilized to smooth each pixel in time sequences without distorting the PT signal intensity. Next, PCA is used to decompose thermography data into principal features, which are fed ICA implemented as a two-layer neural networks structure. The ICA is optimized with fast fixed-point algorithm [17] to further decompose thermography data to speed up detection. The advantages of STDTSS is data-efficient which requires little computation and memory space for fast processing of images quality control of AM structures. We demonstrate performance of STDTSS algorithm in detection of calibrated imprinted defects in SS316L specimen using PT system based on compact IR camera. 


\section{Pulsed Thermography Imaging System}

In the experimental PT system, data is acquired with a laboratory setup shown in Figure 1. A schematic depiction of the setup is shown in Figure 1(a), while the photograph of the laboratory system is presented in Figure 1(b). A pulse trigger sends a signal to capacitor to discharge in a circuit containing white light flash lamp. The flash lamp source Balcar ASYM 6400 delivers a pulse of $6400 \mathrm{~J} / 2 \mathrm{~ms}$ thermal energy to material surface. Heat transfer then takes place from the heated surface to the interior of the sample, resulting in a continuous decrease of the surface temperature. For better heat absorption, all materials in this study were spray-painted with washable graphite paint. Blackbody radiation from the material is simultaneously recorded with a fast frame megapixel IR camera. The flash lamp is typically positioned at $30 \mathrm{~cm}$ to $50 \mathrm{~cm}$ distance to the specimen under investigation. The exact distance and angle of illumination of the flash lamp are determined experimentally for each specimen. This is decided by positioning the IR camera at the focal length of the lens, and placing the flash lamp such that the IR field of view is not blocked, nor does IR camera block the flash. Distribution of incident thermal pulse on the plate is not uniform because the flash light illuminates the metallic plate at an angle.

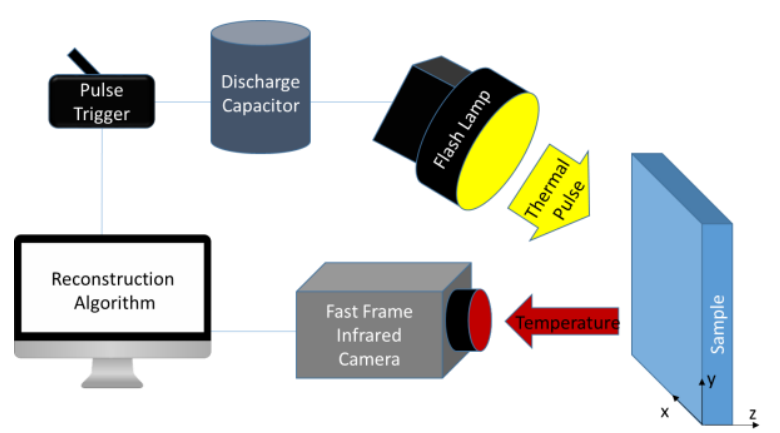

(a)

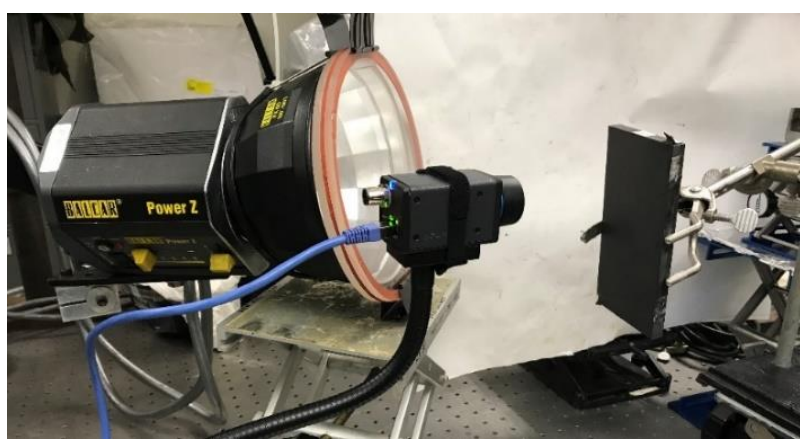

(b)

Figure 1 - Flash thermography experimental setup: (a) Schematic drawing (b) Photograph of actual laboratory system using compact A65 FLIR camera.

A megapixel fast frame infrared (IR) camera records time-resolved images of surface temperature distribution $\mathrm{T}(\mathrm{x}, \mathrm{y}, \mathrm{t})$. The acquired thermal-imaging data cube therefore consist of a series of $2 \mathrm{D}$ images of the sample's surface temperature at consecutive time instants. The laboratory system shown in Figure 17 uses a compact low-cost A65 FLIR camera, which has dimensions ( $\mathrm{L} \times \mathrm{W} \times \mathrm{H})$ of $4.1 \times 1.9 \times 1.8$ in $(295 \times 200 \times 105 \mathrm{~mm})$. The detector is a focal plane array (FPA) uncooled Vanadium Oxide (VOX) microbolometer, which operates in the spectral range $7.5-13 \mu \mathrm{m}$ with noise equivalent temperature detection (NETD) of $50 \mathrm{mK}$ and $12 \mathrm{~ms}$ time constant. The camera has spatial resolution of 640 x 512 pixels with $30 \mathrm{~Hz}$ imaging frame rate. The focal length of the camera lens is $13 \mathrm{~mm}$, resulting in imaging resolution of approximately $140 \mu \mathrm{m}$ per pixel. The total area of the image is $9 \mathrm{~cm} \times 7.2 \mathrm{~cm}$. Performance specifications of A65 FLIR camera are inferior to those of cooled semiconductor IR cameras, such as FLIR indium antimonide 
(InSb) detector $\mathrm{x} 8501 \mathrm{sc}$ camera. The latter has detector integration time of 270ns, NETD sensitivity of $<30 \mathrm{mK}$, and frame rate of at least $180 \mathrm{~Hz}$. Note that in future designs, a flash lamp in Figure 1 could be replaced with a smaller size flash lamp or a laser to make the entire system compact.

Thermography images obtained with a compact IR cameras have lower quality, as compared to those obtained with high-end cooled semiconductor IR camera, because of lower sensitivity, lower sampling rate, and strong noises of small IR camera. As heat deposited with flash lamp the surface starts diffusing into the material bulk, presence of low-density internal material inclusions is revealed through appearance of local temperature "hot spots" on the surface. This effect occurs because low density defects have lower thermal diffusivity compared with solid material. The defects act as thermal resistances and slow down thermal diffusion, which causes temperature difference on the material surface between regions of defects and non-defects. The feature of temperature difference on material surface is used for internal defects detection. However, the intensity of the feature can distorted due to thermal imaging noises, uneven heating of specimens, and low sampling rate of an inexpensive IR camera. 


\section{Sparse Coding with Discrete Cosine Transform (SC/DCT) Algorithm for Removal of Additive White Gaussian Noise from Thermal Images}

While high-resolution thermal images can be obtained from cooled semiconductor detector infrared (IR) cameras, there exist less expensive and smaller form factor microbolometer detector IR cameras. Thermal images obtained with a compact IR camera have lower quality, as compared to those obtained with high-end cooled semiconductor IR camera, because of lower sensitivity, lower sampling rate, and strong noises of small IR camera. Reduction in image quality can be partially compensated with machine-learning based image de-noising algorithms [5]. We model IR camera noises using Additive white Gaussian noise (AWGN) model. In principle, AWGN can be removed from images with a Gaussian filter, but this leads to image blurring. In prior work, we have shown that that quality of images can be enhanced using unsupervised machine learning (ML) Sparse Coding (SC) and K-means Singular Value Decomposition (K-SVD) ML algorithms, which remove AWGN without blurring the images [5]. In this work, we show that the quality of images is enhanced using Sparse Ccoding and Discrete Cosine Transform (SC/DCT) ML algorithm, which removes AWGN without blurring the images.

SC method aims to find the sparse representation of data by searching for the optimized basis vectors of dictionary. In SC method, the dictionary includes the optimized basis vectors called atoms, which are linearly combined to represent image information. Compared to the commonly used Principal Component Analysis (PCA) method, SC allows more flexibility in images denoising as the atoms are not required to be orthogonal. Compared to deep learning methods, such as convolutional neural networks $(\mathrm{CNN})$, training SC model requires less time and fewer computations. DCT, which is a widely used transformation technique, expresses a finite sequence of data points as a sum of cosine functions at different frequencies.

Similar to prior work, SC/DCT approach was applied to TT reconstructions of 3D spatial effusivity e $(\mathrm{x}, \mathrm{y}, \mathrm{z})[5]$. Performance of the SC/DCT approach is demonstrated through imaging of an Inconel 718 nozzle plate fabricated with LPBF method. The plate is $17 \mathrm{~mm}$ thick, and the imaged area is approximately $90 \mathrm{~mm} \times 60 \mathrm{~mm}$. Figure 2 shows a photograph of a laboratory system using high-end FLIR x8501sc camera, and the image of reconstructed effusivity e(x,y) in the plane at $\mathrm{z}=1 \mathrm{~mm}$ depth. Figure 3 shows a photogram of a laboratory system using a compact lower-cost FLIR A65 camera, and corresponding image of reconstructed effusivity e(x,y) in the plane at $\mathrm{z}=1 \mathrm{~mm}$ depth. Image of reconstructed effusivity in Figure 3 is processed with SC/DCT algorithm to remove AWGN. While imaging quality in Figure 3 is lower than that of Figure 2, essential features of the AM structure can be seen in the image in Figure 2. 

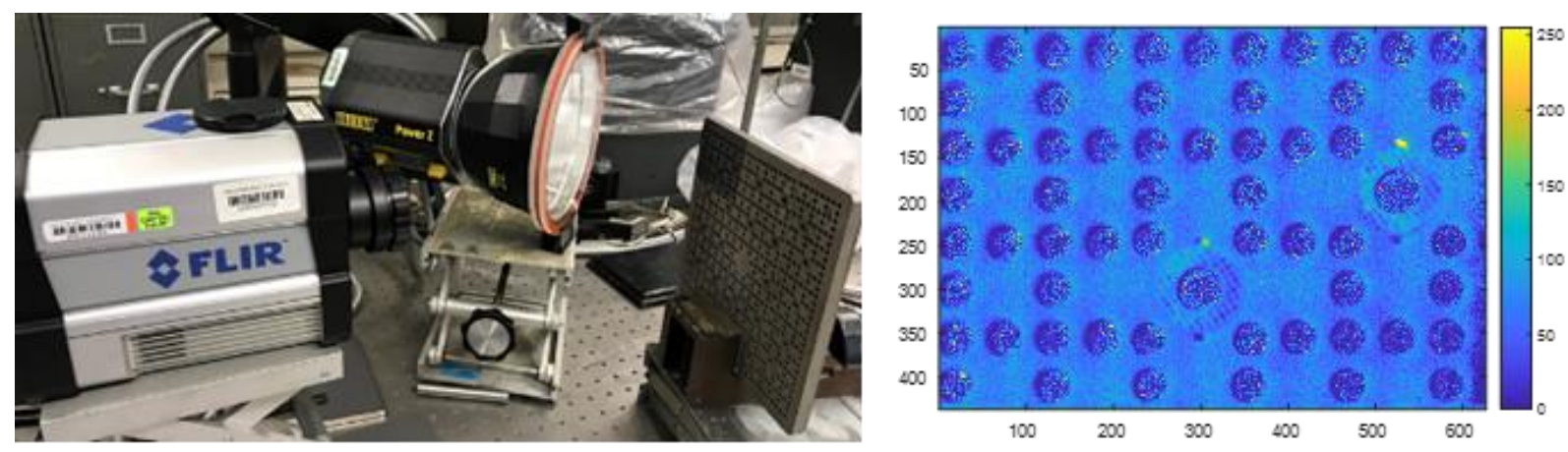

Figure 2 - (Left) Laboratory PTT system using high-end IR camera. (Right) Reconstructed effusivity $\mathrm{e}(\mathrm{x}, \mathrm{y})$ at $\mathrm{z}=1 \mathrm{~mm}$ depth
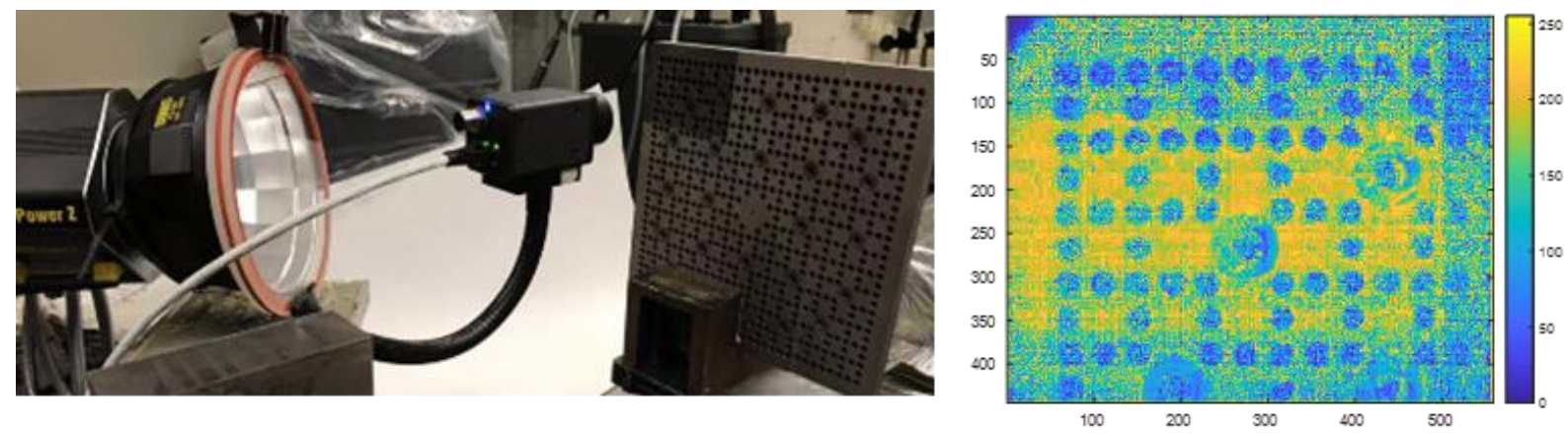

Figure 3 - (Left) Laboratory PTT system using compact lower-cost IR camera. (Right) Reconstructed effusivity $\mathrm{e}(\mathrm{x}, \mathrm{y})$ at $\mathrm{z}=1 \mathrm{~mm}$ depth processed with SC/DCS algorithm. 


\section{Spatial Temporal Denoised Thermal Source Separation (STDTSS) Machine Learning Algorithm}

Thermography images obtained with a compact IR cameras have lower quality, as compared to those obtained with high-end cooled semiconductor IR camera, because of lower sensitivity, lower sampling rate, and strong noises of small IR camera. The objective of this work is to demonstrate that STDTSS compensates for PT image artifacts to enable detection of flaws [14]. The STDTSS algorithm is partly based on previously developed NLBSS algorithm, which was used in PT image analysis in prior work [8]. Because of stronger imaging noises and lower sampling rate of the compact IR camera, we incorporate a number of image denoising features into the STDTSS algorithm. In particular, we design a Gaussian spatial filter and a 7-point Savitzky-Golay temporal filter as preprocessing steps to remove IR imaging noises in space and time. Next, PCA is used to decompose thermography data into principal features, which are fed into ICA implemented as a two-layer neural network structure. The ICA aims to classify and separate the thermography source signals, which correspond to image regions of defects, non-defects, and noise. Each thermography source signal exhibits different temperature evolution during the transient response recorded with PT system. We reconstruct the Thermal Source Image (TSI) from STDTSS to display image regions of defects to detect flaws.

The flow chart of STDTSS algorithm is shown in Figure 4. According to the flow chart, the observed thermograms $X$ are mixed with different thermography source signals which need to be denoised and separated. The Gaussian low pass filter followed by the Savitzky-Golay filter are used to remove noises from thermograms $X$ in space and time. Next, the denoised thermography data cube $\mathrm{X}^{\prime}$ is decomposed with PCA which is implemented as Singular Value Decomposition (SVD) to obtain principal thermography features $U$. The principal components $U$ are fed as inputs to the neural learning-based ICA for further decomposition and classification to estimate the thermal source signals of defects $\mathrm{S}_{e}$, which are TSI of defects. Here W is the separation matrix used to estimate the TSI. 


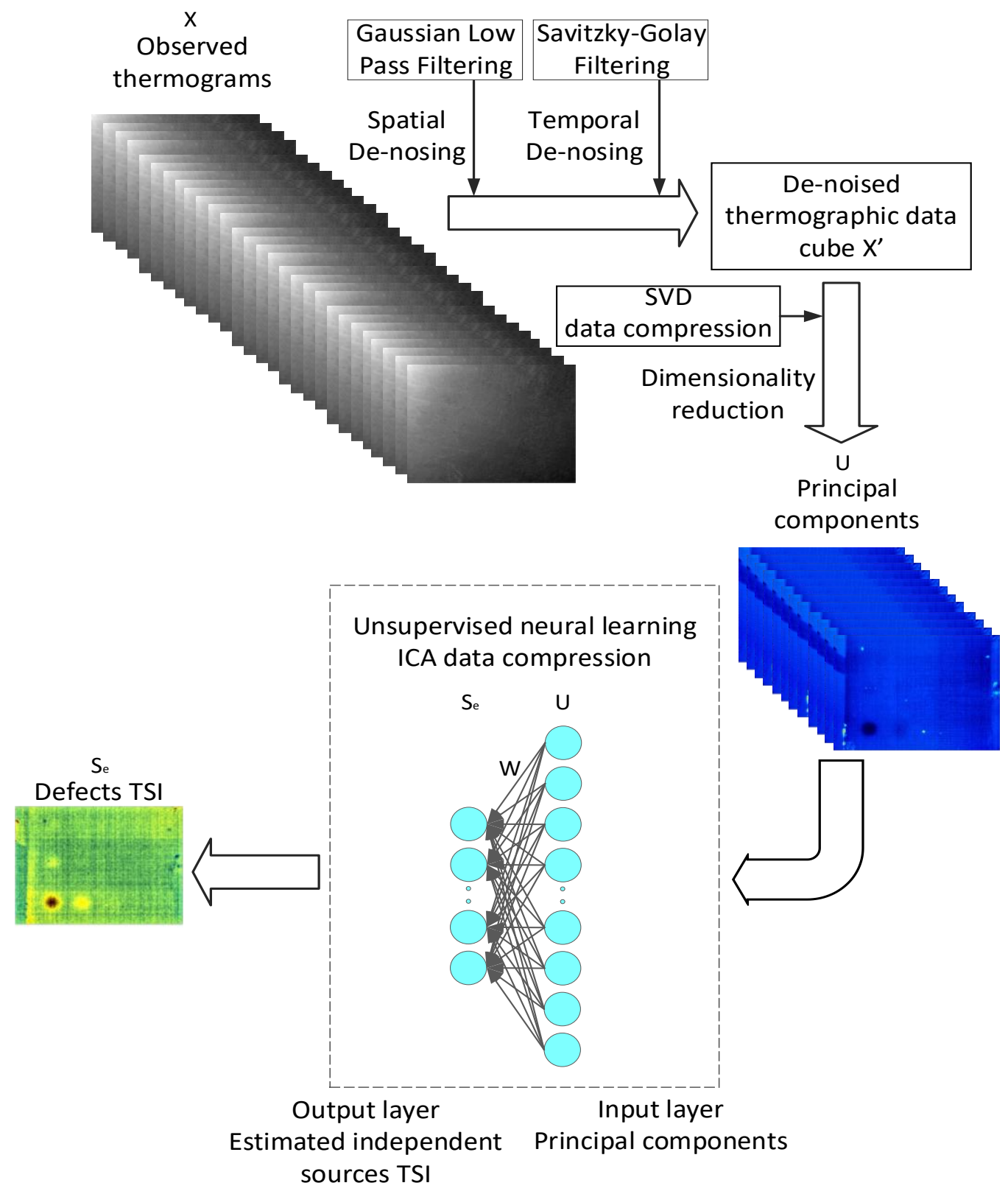

Figure 4 - Flowchart of STDTSS algorithm.

\subsection{Gaussian spatial denoising}

We designed a 3x3 Gaussian low pass filter which removes spatial thermography noises. The 2D Gaussian function is defined as

$$
G(x, y)=\frac{1}{2 \pi \sigma^{2}} e^{-\frac{x^{2}+y^{2}}{2 \sigma^{2}}}
$$


A $3 \times 3$ normalized convolution matrix with $\sigma=0.84$ is produced by the Gaussian function in Equation (1). The matrix $\mathrm{H}$ is used to smooth-out the observed thermography images using the convolution process, while preserving the signatures of material defects. The matrix $\mathrm{H}$ is given as:

$$
H=\left[\begin{array}{lll}
0.06 & 0.13 & 0.06 \\
0.13 & 0.25 & 0.13 \\
0.06 & 0.13 & 0.06
\end{array}\right]
$$

To quantify the denoising performance of the Gaussian spatial filter, we calculate the peak signal-to-noise ratio (PSNR):

$$
P S N R=10 \log _{10}\left(\frac{1}{M S E}\right)
$$

Where the MSE stands for the Mean Squared Error between the observed thermography image and Gaussian denoised thermography image. Using Equation (3) for observed thermography images and Gaussian denoised thermography images, we obtain an enhancement of $\mathrm{PSNR}=40.72 \mathrm{~dB}$.

\subsection{Savitzky-Golay temporal denoising}

Savitzky-Golay filter is a digital filter using selected convolution coefficients for smoothing the temporal data [14]. In PT, each pixel in thermography image acts as a temporal signal and consists of a set of points $\left(x_{j}, y_{j}\right)$, where $x_{j}$ represents the $j^{\text {th }}$ time instant and $y_{j}$ represents the corresponding pixel intensity. These points are noisy in time domain due to thermal imaging noises [10], but are smoothed by a group of $m$ convolutional coefficients $C_{i}$ using the following:

$$
Y_{j}=\sum_{i=\frac{1-m}{2}}^{\frac{m-1}{2}} C_{i} y_{j+i}
$$

Where $\frac{n-1}{2} \leq j \leq n-\frac{1-m}{2}$, and where $n$ is the number of observed thermography images. In this paper, we use the 7-point $(m=7)$ quadratic polynomial convolution coefficients $(-2 / 21,3 / 21,6 / 21$, $7 / 21,-2 / 21,3 / 21,6 / 21)[14]$ to enhance the precision of data without distorting the PT signal.

\subsection{Neural learning-based matrix decomposition}

To detect flaws, the denoised thermography data cube $X^{\prime}$ is decomposed with SVD to extract principal features $(U)$ of defects by removing non-relevant information, such as un-even heating due to flash lamp pulse incident at an angle on the material surface (see Figure 1). Next, the principal features are used as inputs to train the neural learning-based ICA to separate image regions containing signatures of defects from image regions do not contain any material defects. After the separation matrix $W$ is trained using ICA, this matix is applied to detect flaws by 
separating the mixed thermograhms into TSIs of defects. This neural learning-based ICA consists of one input layer (principal features $U$ ), and one output layer (estimated source signal $S_{e}$ ). The input layer has 20 neurons, equal to the number of principal components. The output layer has 8 neurons, equal to the number of independent components. The nonlinearity function "pow3" $\left(g(u)=u^{3}\right)$ [14] is used as the activation funtion to optimize training with fast fixed-point algorithm [15] for robust covergence. In addition, the reverse entropy [15] is applied as the objective funciton to measure the non-Gaussian. We train this two-layer neural network with 100 epochs to estimate TSI of defects. 


\section{Detection of Imprinted Calibrated Defects in SS316L AM Plate with STDTSS Algorithm}

\subsection{Development of SS316L additively manufactured specimens}

Calibrated defects with hemispherical shape were imprinted into SS316L specimen to study STDTSS algorithm performance in detection of material flaws. While pores in AM metals can have a variety of shapes, the spherical shape of the calibrated defect provides a generic representation of material flaw. For studying PT performance, either hemispherical or spherical shape defects provide similar target characteristics. A stainless steel SS316L plate was fabricated with direct metal laser melting (DMLM) method using EOS metal 3D printer (GPI Prototyping and Manufacturing). DMLM, also known as direct metal laser sintering (DMLS) is one of the methods in the LPBF category of AM processes. Fabrication of SS316L plate was based on $20 \mu \mathrm{m}$ powder layers. The dimensions of the plate are Length $\times$ Width $\times$ Thickness: 6 in $\times 3$ in $\times 0.4$ in $(152 \mathrm{~mm} \times 76 \mathrm{~mm} \times 10 \mathrm{~mm})$. A pattern of calibrated defects consisting of hemispherical porosity regions containing un-sintered metallic powder were imprinted into the SS316L plates. The defects were imprinted into the plates during fabrication using an STL (sterelithography) file with a drawing of the pattern of hemispherical inclusions [4].
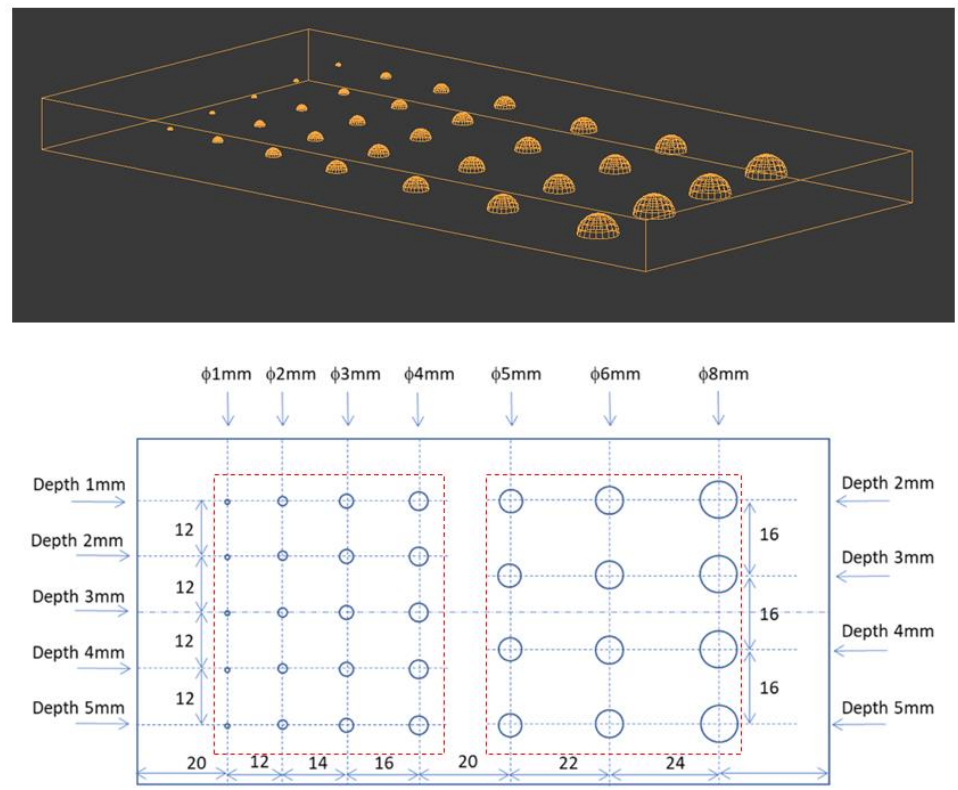

Figure 5 - (Top) 3D rendering of hemispherical porosity defects imprinted in AM specimens. (Bottom) Design pattern of hemispherical defects of different diameters and depths relative to plate surface (all dimensions are in $\mathrm{mm}$ )

A computer rendering of the pattern of defects is shown in Figure 5 (top panel). A drawing with labels showing diameters and depths of the imprinted defects is provided in Figure 5 (bottom panel). Note that there are two patterns of defects on the plate: one with diameters $\phi=5,6$ and $8 \mathrm{~mm}$ and depths $d=2,3,4$, and 5mm, and another one with diameters $\phi=1,2,3$, and $4 \mathrm{~mm}$ and 
depths $d=1,2,3,4$ and $5 \mathrm{~mm}$. The diameters of defects decrease along the lines parallel to the longer side of the plate, while the depth along these lines is held constant. Along the lines parallel to the shorter side of the plate, the depth increases, while the diameter is fixed along these lines.

Using the set up shown in Figure 1, we obtained images of porosity defects imprinted in SS316L plate. Imaging was performed with plate oriented in way that the hemispherical sides of the internal defects were facing the IR camera and flash lamp. Separate measurements were made imaging larger and smaller defects patterns.

\subsection{Detection of larger defects with STDTSS algorithm}

In the first experiment, PT imaging was performed on the pattern with larger defects of the AM SS316L plate, which is shown with the red box in Figure 6(a). Figures 6(b) and 6(c) show the observed thermography frame and the estimated TSI of defects from STDTSS, respectively. The red dashed line oval in Figure 6(a) indicates which of the imprinted defects were detected. Defects $(\varnothing 8, \mathrm{~d} 2),(\varnothing 8, \mathrm{~d} 3)$, and $(\varnothing 6, \mathrm{~d} 2)$ can be clearly seen, while defects $(\emptyset 8, \mathrm{~d} 4),(\varnothing 6, \mathrm{~d} 3)$, and $(\varnothing 5, \mathrm{~d} 2)$ are detected with relatively lower confidence.

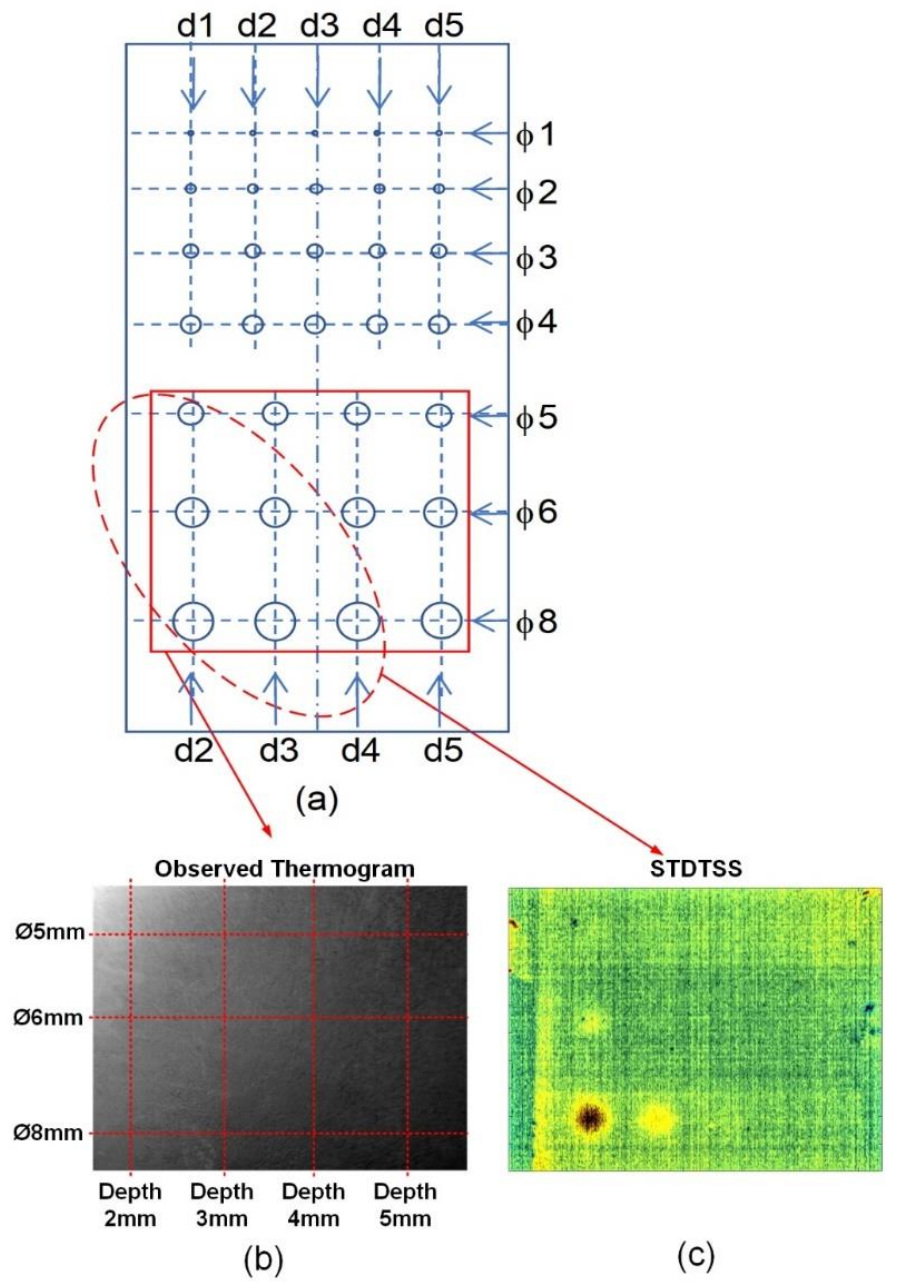

Figure 6 - Results of detection of larger defects in SS316L specimen. (a) Area of the plate (b) Raw thermogram (c) Estimated TSI with STDTSS. 


\subsection{Detection of smaller defects with STDTSS algorithm}

In the second experiment, we repeated PT measurements with the smaller pattern of defects imprinted into the AM SS316L plate. The area of the plate is shown in Figure 7(a). The red dashed line oval in Figure 7(a) indicates which of the imprinted defects were detected. Figures 7(b) and 7(c) show the observed thermography frame and the estimated TSI of defects from STDTSS, respectively. Defects $(\varnothing 4, \mathrm{~d} 1)$ and $(\varnothing 3, \mathrm{~d} 1)$ can be clearly seen, while defects $(\varnothing 2, \mathrm{~d} 1)$ and $(\varnothing 1$, d1) are detected with relatively lower confidence.

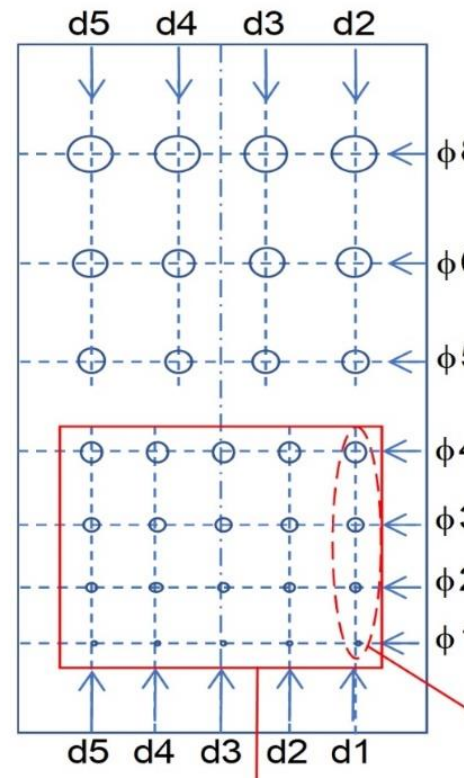

(a)

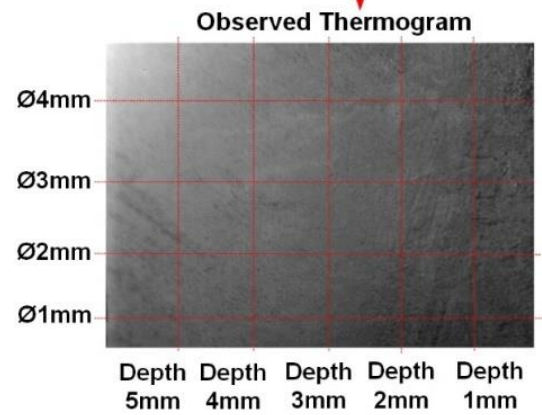

(b)

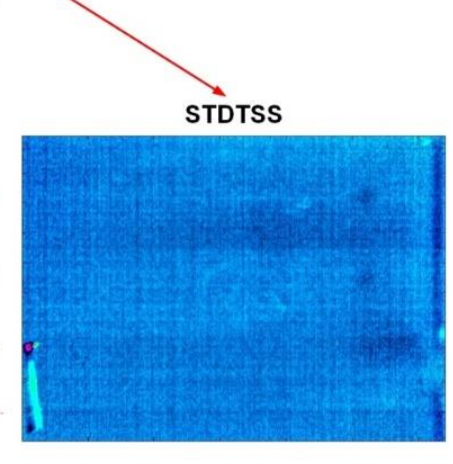

(c)

Figure 7 - Results of detection of smaller defects in specimen SS316L specimen. (a) Area of the plate (b) Raw thermogram (c) Estimated TSI with STDTSS. 


\section{Conclusions}

AM offers the potential of reduced-cost manufacturing of structures for nuclear energy applications. LPBF is a common method for AM of high-strength corrosion-resistant alloys used in nuclear energy application. However, intrinsic features of LPBF process can lead to appearance for porosity defects in AM structures. Quality control of AM structures is needed before their deployment in the harsh environment of a nuclear reactor. Pulsed thermography (PT) method provides non-destructive capability to image actual structures. In this report, we have investigated detection of imprinted regions of porosity defects in AM SS316L specimen using a compact and relatively low-cost IR camera. Using such camera is advantageous for cost-reduction of the manufacturing and qualification process, as well for imaging complex geometry AM structures with spatially constrained access. However, small form factor IR camera, which is typically based on an uncooled microbolometer detector, has lower sensitivity, integration time, and sampling rate compared to high-end cooled semiconductor detector IR camera. This results in higher noise and lower detection sensitivity in thermography images obtained with a compact IR camera.

We have investigated the possibility of using unsupervised ML algorithms to compensate for loss in imaging quality with a compact IR camera, which can be used for in-service NDE applications. In one approach, we have developed SC/DCT algorithm, which efficiently removes AWGN without blurring images. Performance of SC/DCT algorithm was demonstrated through processing of TT images of IN718 AM plate using a compact PT system based on FLIR A65 camera. This compact and relatively low-cost camera uses an uncooled microbolometer detector arrays. Images processed with SC/DCT were compared with those of the same IN718 plate obtained with high-end FLIR x8501 camera, which is based on cooled semiconductor detector.

In another approach, we developed unsupervised ML-based STDTSS algorithm for nondestructive detection of subsurface defects and qualification of AM structures with a compact PT system. In STDTSS, a 2D Gaussian filter is designed to spatially denoise high frequency components in thermography images while preserving signatures of material defects. In addition, a 7-point Savitzky-Golay filter is utilized to smooth each pixel in time sequences without distorting the PT signal intensity. Next, PCA is used to decompose thermography data into principal features, which are fed into ICA implemented as a two-layer neural networks structure. The ICA is optimized with fast fixed-point algorithm to further decompose thermography data to speed up detection. The STDTSS is data-efficient which requires little computation and memory space for fast analysis and qualification of AM structures. Performance of the STDTSS algorithm was demonstrate in imaging of an AM structure with a compact PT system based on small FLIR A65 camera. An AM SS316 plate used in the imaging study contained calibrated internal hemispherical defects with un-sintered powder, which were imprinted into the specimen with LPBF process. To further enhance flaws detection, future work will involve optimizing the STDTSS by utilizing either sparse PCA or non-linear PCA.

Further improvements in thermography hardware and ML algorithms would be needed to detect smaller and deeper-located defects, such as increasing the power of the flash lamp, and using a microscopic lens to increase the number of pixels per an image of a defect could enhance 
detection resolution. In addition, possible improvement of imaging can be obtained by developing more elaborate models of IR camera noises, and training ML to improve their de-noising performance. 


\section{References}

1. X. Lou and D. Gandy, “Advanced Manufacturing for Nuclear Energy,” JOM 71, 2834-2836 (2019).

2. S. A. Khairallah, A. T. Anderson, A. Rubenchik, W. E. King, "Laser Powder-Bed Fusion Additive Manufacturing: Physics of Complex Melt Flow and Formation Mechanisms of Pores, Spatter and Denudation Zones," Acta Materialia 108, 36-45 (2016).

3. M. D. Sangid, P. Ravi, V. Prithivirajan, N. A. Miller, P. Kenesei, J.-S. Park, "ICME Approach to Determining Critical Pore Size of IN718 Produced by Selective Laser Melting," JOM 72, 465-474 (2019).

4. X. Zhang, J. Saniie, W. Cleary, A. Heifetz. (2020). "Quality Control of Additively Manufactured Metallic Structures with Machine Learning of Thermography Images," JOM 72(12), 4682-4694 (2020).

5. X. Zhang, J. Saniie, A. Heifetz, "Detection of Defects in Additively Manufactured Stainless Steel 316L with Compact Infrared Camera and Machine Learning Algorithms," JOM 72(12), 4244-4253 (2020).

6. X. Zhang, B. Wang, J. Saniie, "Deep Convolutional Neural Networks Applied to Ultrasonic Images for Material Texture Recognition," 2020 IEEE International Ultrasonics Symposium (2020).

7. A. Heifetz, X. Zhang, J. Saniie, D. Shribak, T. Elmer, S. Bakhtiari, B. Khaykovich, W. L Cleary, "Second Annual Progress Report on Pulsed Thermal Tomography Nondestructive Examination of Additively Manufactured Reactor Materials," Argonne National Laboratory ANL-20/62 (2020).

8. X. Zhang, J. Saniie, A. Heifetz, "Neural Learning Based Blind Source Separation for Detection of Material Defects in Pulsed Thermography Images," 2020 IEEE International Conference on Electro-Information Technology (EIT), 112-116 (2020).

9. A. Heifetz, D. Shribak, X. Zhang, J. Saniie, Z.L. Fisher, T. Liu, J.G. Sun, T. Elmer, S. Bakhtiari, W. Cleary, "Thermal Tomography 3D Imaging of Additively Manufactured Metallic Structures," AIP Advances 10(10), 105318 (2020).

10. A. Heifetz, J.G. Sun, D. Shribak, T. Liu, T.W. Elmer, P. Kozak, S. Bakhtiari, B. Khaykovich, W. Cleary, "Pulsed Thermal Tomography Nondestructive Evaluation of Additively Manufactured Reactor Structural Materials," Transactions of the American Nuclear Society 121(1), 589-591 (2019).

11. A. Heifetz, D. Shribak, Z.L. Fisher, W. Cleary, "Detection of Defects in Additively Manufactured Metals Using Thermal Tomography,” TMS 2021 150 th $^{\text {th }}$ Annual Meeting \& Exhibition Supplemental Proceedings, 121-127 (2021).

12. E. D'Accardi, F. Palano, R. Tamborrino, D. Palumbo, A. Tatì, R. Terzi, U. Galietti. (2019). "Pulsed Phase Thermography Approach for the Characterization of Delaminations in CFRP and Comparison to Phased Array Ultrasonic Testing," Journal of Nondestructive Evaluation 38,20 (2019). 
13. C. Ibarra-Castanedo, A. Bendada and X. Maldague, "Thermographic Image Processing for NDT," IV Conferencia Panamericana de END, Buenos Aires (2007)

14. X. Zhang, J. Saniie, A. Heifetz, "Spatial Temporal Denoised Thermal Source Separation in Images of Compact Pulsed Thermography System for Qualification of Additively Manufactured Metals," submitted to 2021 IEEE International Conference on ElectroInformation Technology (EIT) (2021).

15. X. Zhang, T. Gonnot, J. Saniie, "Real-Time Face Detection and Recognition in Complex Background," Journal of Signal and Information Processing 8, 99-112 (2017)

16. A. Gorry, "General Least-Squares Smoothing and Dfferentiation by the Convolution (Savitzky-Golay) Method," Analytical Chemistry 62(6), 570-573 (1990).

17. A. Hyvärinen, "The Fixed-Point Algorithm and Maximum Likelihood Estimation for Independent Component Analysis," Neural Processing Letters 10, 1-5 (1999). 


\section{Argonne}

Nuclear Science and Engineering (NSE) Division

Argonne National Laboratory

9700 South Cass Avenue, Bldg. 208

Argonne, IL 60439

www.anl.gov

Argonne National Laboratory is a U.S. Department of Energy laboratory managed by UChicago Argonne, LLC 